\title{
HEAVY HADRON SPECTROSCOPY AND THE BAG MODEL
}

\author{
A. Bernotas and V. Šimonis \\ Vilnius University Institute of Theoretical Physics and Astronomy, A. Goštauto 12, LT-01108 Vilnius, Lithuania \\ E-mail: bernotas@itpa.lt, simonis@itpa.lt
}

Received 29 December 2008; revised 14 March 2009; accepted 19 March 2009

\begin{abstract}
Some time ago a slightly improved variant of bag model (the modified bag model) suitable for the unified description of light and heavy hadrons was developed. The main goal of the present work was to calculate the masses of the ground state baryons containing bottom quarks in the framework of this model. For completeness the predictions for other heavy hadrons are also given. The reasonable agreement of our results with other theoretical calculations and available experimental data suggests that our predictions could serve as a useful complementary tool for the interpretation of heavy hadron spectra.
\end{abstract}

Keywords: bag model, heavy quarks, heavy baryons, hadron spectra

PACS: $12.39 . \mathrm{Ba}, 14.20 . \mathrm{Lq}, 14.20 . \mathrm{Mr}, 14.40 . \mathrm{Lb}, 14.40 . \mathrm{Nd}$

\section{Introduction}

During the last decade a significant progress has been achieved in the experimental and theoretical studies of heavy hadrons (for review see [1]). In the nearest future a considerable amount of new experimental data in the bottom (beauty) sector is expected from the Large Hadron Collider (LHC). Therefore the theoretical calculation of the masses of heavy baryons containing bottom quarks becomes more urgent. Ideally, one would like to obtain the hadron masses from the first principles, say, performing the nonperturbative lattice QCD calculations. But the available quenched lattice predictions for heavy hadron masses [2-6] still suffer from rather large numerical uncertainties due to finite size effects, systematic and statistical errors. The further progress in lattice QCD is connected with improved calculations which take account of degrees of freedom associated with the light (up, down, and strange) sea quarks. Some preliminary results there already exist [7], and impressive success of the lattice QCD in the heavy hadron sector is the prediction of mass of the $B_{c}$ meson [8]. Despite the lack of high accuracy, even the so far available lattice calculations are of crucial importance for the consistent treatment of the heavy hadron properties. First of all, they serve as a good starting point for the further analysis of heavy hadron spectrum. On the other hand, if one is interested only in calculating the mass splittings, then major uncertainties cancel out and the accuracy of lattice predictions increases.
Of course, there are other methods available: QCD sum rules, heavy quark effective theory, various potential models, bag model, etc. As a rule, they are based on some assumptions and approximations. However, taken all together, they have become a powerful tool capable to give reasonable predictions for the heavy hadron properties. QCD sum rules is another (besides lattice QCD) nonperturbative approach which could be applied to study heavy baryon spectrum. Treatment based on this approach $[9,10]$ provides the results consistent with experimental observations, yet one must not expect very high precision in this case. Another very fruitful and interesting approach is based on the expansion in inverse powers of the heavy quark mass. The effective heavy quark theory obtained in this way has become rather popular tool in heavy hadron physics and a new approximate heavy quark symmetry has been discovered (for review see [11-14]. Various potential models are widely used and generally regarded as being rather successful. Indeed, it is amazing how well the potential model predictions fit large and varied data set. Even the early treatments [15-17] predict the masses of many hadrons with good accuracy. Among the recent treatments there are extensive developments of the traditional approach $[18,19]$, calculations using the method of hyperspherical harmonics [20], solving the Faddeev equations [21], variational calculations [22-24], nonrelativistic calculations using quark-diquark approximation [25,26], etc. Relativistic effects are taken into account by building up the 
relativistic quark models based on approximate solution of the Bethe-Salpeter equation [27], or using quarkdiquark approximation to simplify relativistic dynamics [28-30]. Almost all approaches give predictions in fair agreement with data, at least in the charm sector. This would seem to imply that there must be a truthful basis in all this machinery. However, one should not forget that the potential models are essentially of a phenomenological nature. The interaction built into a particular model is in fact the effective interaction, therefore there must be no surprise that sometimes different approaches give similar results and vice versa. One possible and widely used form of the interaction, proposed in the seminal work [31], imitates the one-gluon exchange. Other examples of the effective interactions used in the heavy hadron spectroscopy are: the phenomenological extension of the instanton-induced force [27] and the flavour dependent interactions suggested by the Goldstone-boson exchange [20,32,33]. Some mixture of one-gluon-exchange and Goldstone-bosonexchange induced interactions is also possible $[22,34]$. The bag model was originally designed for the ultrarelativistic case of the light quarks [35]. The first straightforward application of the model to calculate the spectrum of the hadrons containing heavy quarks was not very successful [36]. Later on, there were several attempts to calculate the spectrum of the heavy hadrons using improved versions of the model $[37,38]$. Another improved version of the bag model suitable for the unified description of light and heavy hadrons was proposed by present authors in the Ref. [39]. Now we present our predictions for the masses of the ground state baryons containing bottom quarks that are calculated using this version of the model (the modified bag model).

The paper is organized as follows: Section 2 very briefly describes the modified bag model. For more details we refer to [39]. In the beginning of Section 3 we present our previous calculations (with some minor improvements) for the charm sector baryons and for the heavy mesons belonging to the charm and bottom sectors. Our results are compared with the estimates obtained in various other approaches and with experimental data where available. This may be regarded as a test of the model and gives us some feeling of what should be expected in the case of the bottom baryons. Then the modified bag model predictions for the ground state baryons containing bottom quarks are presented and discussed. This is the main result of the present paper. Finally, in Section 4 we conclude with the con- siderations on the role of the bag model in the hadron spectroscopy.

\section{Modified bag model}

The calculation of the ground state hadron mass proceeds in two steps. First, the so-called bag energy associated with particular hadron is calculated. It depends on the bag radius $R$ and is defined by

$$
E=E_{V}+E_{Q}+E_{M}+E_{C},
$$

where $E_{V}$ is the bag volume energy,

$$
E_{V}=\frac{4 \pi}{3} B R^{3},
$$

and $E_{Q}$ is the sum of single-particle quark energies,

$$
E_{Q}=\sum_{i} n_{i} \varepsilon_{i} .
$$

In order to determine the numerical values of eigenenergies $\varepsilon_{i}$ the free Dirac equation is solved for each quark subject to linear boundary condition that ensures the vanishing of all vector currents at the bag surface. $E_{M}$ and $E_{C}$ are the colour-magnetic and colour-electric (Coulomb) interaction energies. They can be calculated explicitly [39]. The numerical value of the bag energy is obtained minimizing (1) as a function of $R$.

The quarks in the bag are not in an eigenstate of the total momentum. A part of the bag energy is spurious and comes from the motion of the centre-of-mass (c.m.m. problem). In order to obtain the mass of the hadron it is necessary to incorporate the c.m.m. corrections, in some way. We follow the Ref. [40] and assume the relation between the calculated bag-model energy $E$ and the mass $M$ of particular hadron to be

$$
E=\int \mathrm{d}^{3} s \Phi_{P}^{2}(s) \sqrt{M^{2}+s^{2}},
$$

where $\Phi_{P}(s)$ is a Gauss profile,

$$
\Phi_{P}(s)=\left(\frac{3}{2 \pi P^{2}}\right)^{3 / 4} \exp \left(-\frac{3 s^{2}}{4 P^{2}}\right) .
$$

The effective momentum $P$ which specifies the momentum distribution is defined by

$$
P^{2}=\gamma \sum_{i} n_{i} p_{i}^{2}
$$

where $p_{i}$ are the momenta of the individual quarks and $\gamma$ is the model parameter governing the c.m.m. correction. 
Table 1. Parameters (in GeV) used to determine the behaviour of the mass functions $\bar{m}_{f}(R)$.

\begin{tabular}{cccc}
\hline$f$ & $s$ & $c$ & $b$ \\
\hline$\widetilde{m}_{f}$ & 0.161 & 1.458 & 4.793 \\
$\delta_{f}$ & 0.156 & 0.112 & 0.061 \\
\hline
\end{tabular}

In order to obtain the mass of the particle, Eq. (4) is to be solved numerically. However, in the presence of bottom quarks this equation is practically equivalent to the relation [40]

$$
M^{2}=E^{2}-P^{2},
$$

and in this case we prefer to use this simple relation instead of the rather cumbersome procedure based on Eq. (4).

The interaction energies are computed to the first order in the scale-dependent strong coupling constant $\alpha_{\mathrm{c}}(R)$, and for the strange, charmed, and bottom quarks we use the running mass $\bar{m}_{f}(R)$ [39]. The light (up and down in our case) quarks are assumed to be massless.

For the running coupling constant we use the expression proposed by Donoghue and Johnson [41]

$$
\alpha_{\mathrm{c}}(R)=\frac{2 \pi}{9 \ln \left(A+R_{0} / R\right)},
$$

where $R_{0}$ is the scale parameter which plays the role similar to QCD constant $\Lambda$. The model parameter $A$ serves to avoid divergences in the case when $R \rightarrow R_{0}$.

For the mass function $\bar{m}_{f}(R)$ we use the expression

$$
\bar{m}_{f}(R)=\widetilde{m}_{f}+\alpha_{\mathrm{c}}(R) \cdot \delta_{f},
$$

thus, for each quark flavour there are two free model parameters $\widetilde{m}_{f}$ and $\delta_{f}$.

Altogether we have ten free parameters in the model. The four of them $\left(B, \gamma, A\right.$, and $\left.R_{0}\right)$ were determined by fitting calculated masses of light hadrons $(N, \Delta, \pi$, and the average mass of the $\omega-\rho$ system) to experimental data. The numerical values of these parameters are the same as in the Ref. [39]: $B=7.597 \cdot 10^{-4} \mathrm{GeV}^{4}$, $R_{0}=2.543 \mathrm{GeV}^{-1}, A=1.070, \gamma=1.958$. In order to fix the remaining six parameters $\left(\widetilde{m}_{s}, \delta_{s}, \widetilde{m}_{c}, \delta_{c}, \widetilde{m}_{b}, \delta_{b}\right)$ necessary to define the mass functions $\bar{m}_{f}(R)$ we have employed the masses of vector mesons $(\phi, J / \psi, \Upsilon)$ accompanied by the mass values of the lightest baryons $\Lambda_{f}$ belonging to the corresponding flavour sector. The results are presented in Table 1.

The numerical values of the parameters $\widetilde{m}_{c}, \delta_{c}, \widetilde{m}_{b}$, and $\delta_{b}$ differ slightly from the corresponding values adopted in [39] because in the present work we have used new, more accurate mass values of $\Lambda_{c}(2.286 \mathrm{GeV})$ and $\Lambda_{b}(5.620 \mathrm{GeV})[42]$.

We end up this section with the remark about the baryon mixing problem. It is well known that the hyperfine interaction mixes the wave functions of ground state spin- $1 / 2$ baryons containing three quarks of different flavours [43]. In our case such baryons are $\Xi_{c}$ and $\Xi_{c}^{\prime}, \Xi_{b}$ and $\Xi_{b}^{\prime}, \Xi_{b c}$ and $\Xi_{b c}^{\prime}, \Omega_{b c}$ and $\Omega_{b c}^{\prime}$. In order to avoid ambiguities associated with the ordering of the quarks in the wave function $\left|\left(q_{1} q_{2}\right)^{S} q_{3}\right\rangle$ we simply calculate the off-diagonal elements of the interaction energy matrix with consequent matrix diagonalization (for details see [44]). The alternative choice would be to use the so-called optimal basis by picking up the heaviest quark as the third one in the spin coupling scheme $\left(q_{1} q_{2}\right)^{S} q_{3}[43,44]$. For the c.m.m. uncorrected bag energies both choices give practically identical results. The resulting mass values may differ slightly. This is so, because the spurious c.m.m. energy, which must be subtracted from the hadron energy, depends on the hadron bag radius $R_{H}$. When we are dealing with a mixed set of wave functions, we minimize the trace of the energy matrix, $E_{B}+E_{B^{\prime}}$, which remains invariant under state mixing. So, in this case the bag radii for both mixed hadrons coincide. On the other hand, if the optimal basis is used, the natural choice would be to minimize the energy of each baryon individually. Then the radii of baryons under consideration are no longer identical (practically the difference is quite small). The shift in mass caused by such change of bag radii does not exceed $2 \mathrm{MeV}$, which is obviously smaller than the systematic uncertainties of the model and, in principle, has nothing to do with the baryon mixing.

\section{Predictions for the ground state hadron masses}

Strictly speaking, the bag model is not derivable from QCD and the quality of its predictions is not quite clear. We can only compare the results of calculations with experimental data (if available) as well as with calculations using various other approaches. A good starting point for such comparison ought to be the charm sector. As a first step let us compare our predictions for the hadrons from this sector with the results obtained in other variants of the bag model. The results for hadron masses and mass splittings are presented in Tables 2 and 3 respectively. The columns of the tables denoted as MIT contain the original results from the Ref. [36]. In Ref. [45] the quark-quark interaction is treated perturbatively. Moreover, in this work the nonphysical selfenergy term (which was present in the original MIT ver- 
Table 2. Masses (in $\mathrm{GeV}$ ) of ground state hadrons from the charm sector, calculated in five variants of the bag model as described in the text. The column denoted as Exp contains averaged over isomultiplets experimental data.

\begin{tabular}{clllllll}
\hline Hadrons & Quarks & Exp & Our & MIT & {$[45]$} & {$[37]$} & {$[38]$} \\
\hline$J / \psi$ & $c \bar{c}$ & 3.097 & 3.097 & 3.095 & 3.15 & 3.095 & - \\
$\eta_{c}$ & $c \bar{c}$ & 2.980 & 3.005 & 2.931 & 3.05 & 2.971 & - \\
$D$ & $c \bar{u}$ & 1.867 & 1.834 & 1.726 & 1.82 & 1.800 & 1.83 \\
$D^{*}$ & $c \bar{u}$ & 2.008 & 2.002 & 1.969 & 2.01 & 2.009 & 2.01 \\
$D_{s}$ & $c \bar{s}$ & 1.968 & 1.965 & 1.885 & 1.98 & 1.957 & 1.92 \\
$D_{s}^{*}$ & $c \bar{s}$ & 2.112 & 2.119 & 2.099 & 2.14 & 2.141 & 2.09 \\
$\Lambda_{c}$ & $c u u$ & 2.286 & 2.286 & 2.214 & 2.29 & 2.243 & 2.28 \\
$\Sigma_{c}$ & $c u u$ & 2.454 & 2.393 & 2.357 & 2.42 & 2.380 & 2.38 \\
$\Sigma_{c}^{*}$ & $c u u$ & 2.518 & 2.489 & 2.461 & 2.53 & 2.481 & 2.49 \\
$\Xi_{c}$ & $c s u$ & 2.469 & 2.468 & 2.396 & 2.48 & 2.425 & 2.43 \\
$\Xi_{c}^{\prime}$ & $c s u$ & 2.577 & 2.546 & 2.507 & - & 2.530 & 2.50 \\
$\Xi_{c}^{*}$ & $c s u$ & 2.646 & 2.638 & 2.603 & 2.67 & 2.624 & 2.60 \\
$\Omega_{c}$ & $c s s$ & 2.697 & 2.697 & 2.653 & 2.73 & 2.678 & 2.61 \\
$\Omega_{c}^{*}$ & $c s s$ & 2.768 & 2.783 & 2.742 & - & 2.764 & 2.71 \\
$\Xi_{c c}$ & $c c u$ & 3.519 & 3.557 & 3.538 & 3.66 & 3.511 & - \\
$\Xi_{c c}^{*}$ & $c c u$ & - & 3.661 & 3.661 & - & 3.630 & - \\
$\Omega_{c c}$ & $c c s$ & - & 3.710 & 3.690 & 3.82 & 3.664 & - \\
$\Omega_{c c}^{*}$ & $c c s$ & - & 3.800 & 3.795 & - & 3.764 & - \\
$\Omega_{c c c}$ & $c c c$ & - & 4.777 & 4.827 & 4.98 & 4.747 & - \\
\cline { 3 - 8 } & & - & 0.023 & 0.067 & 0.03 & 0.036 & 0.05 \\
\hline
\end{tabular}

sion of the model) is omitted. In Ref. [37] the improvement of the hadronic mass spectrum is achieved introducing some extra dependence of the bag energy on the heaviest (inside the hadron) quark mass. The variant of model used in Ref. [38] was constructed for hadrons containing one heavy quark. In this approach the heavy quark is treated as a point source of colour fields located at the centre of the bag. The experimental values are taken from the Particle Data Tables [42]. For the isospin multiplets the averaged values are used here and further on. To show the quark content of the hadrons we use the symbols $b, c, s$ for the bottom, charmed, and strange quarks, respectively. For the sake of simplicity the symbol $u$ is used for both light (up or down) quarks. The corresponding antiquarks are denoted as $\bar{b}$, $\bar{c}, \bar{s}$, and $\bar{u}$. In the last row of the Table 2 the values of the root mean squared deviation from the experimenal mass spectra

$$
\chi=\left[\frac{1}{N} \sum_{i=1}^{N}\left(M^{i}-M_{\mathrm{ex}}^{i}\right)^{2}\right]^{1 / 2}
$$

are presented. The mass of the $\Xi_{c c}$ baryon has not been included in the summation.

We see from Table 2 that the original MIT results are in serious conflict with experimental data and discrepancies seem to be of systematic character. The improved variants [37], [38], and [45] give evidently more reasonable predictions. The agreement of our predictions with available experimental data is rather good, although there are some discrepancies. The most serious problem common to almost all variants of bag model seems to be the mass of $\Sigma_{c}$ baryon (its analog $\Sigma$ in the sector of light hadrons was also problematic [39]). One possible way to improve the description of these states would be the inclusion of chiral (pionic) corrections [46-48], however, such extension is beyond the scope of the present investigation.

Let us refer to the Table 3. There we compare our predictions for some hadron mass splittings with experimental data and other bag model calculations. For the baryons all variants of the bag model give similar predictions about $30 \%$ larger than needed. This may indicate that the interaction strength for baryons in the bag model is somewhat overestimated, even in the versions of the model where the running coupling constant is used. In the meson sector our predictions for the $D^{*}-D$ and $D_{s}^{*}-D_{s}$ mass splittings are better than in other approaches, though still somewhat larger than experimental values. For the $J / \psi-\eta_{c}$ we together with Ref. [45] predict somewhat smaller than required mass difference. In general, we see that the overall agreement of our predictions with available experimental data is good ( $\Sigma_{c}$ mass being the exception). Moreover, as a rule, our predictions almost in all cases are better than the predictions given by other variants of the bag model. 
Table 3. Mass splittings (in $\mathrm{GeV}$ ) of some hadrons from the charm sector, calculated in five variants of the bag model as described in the text. The column denoted as Exp contains experimental data.

\begin{tabular}{lllllll}
\hline Hadrons & Exp & Our & MIT & {$[45]$} & {$[37]$} & {$[38]$} \\
\hline$J / \psi-\eta_{c}$ & 0.117 & 0.092 & 0.164 & 0.10 & 0.124 & - \\
$D^{*}-D$ & 0.141 & 0.168 & 0.244 & 0.19 & 0.209 & 0.18 \\
$D_{s}^{*}-D_{s}$ & 0.144 & 0.154 & 0.214 & 0.16 & 0.184 & 0.17 \\
$\Sigma_{c}^{*}-\Sigma_{c}$ & 0.064 & 0.096 & 0.104 & 0.11 & 0.101 & 0.11 \\
$\Xi_{c}^{*}-\Xi_{c}^{\prime}$ & 0.069 & 0.092 & 0.096 & - & 0.094 & 0.10 \\
$\Omega_{c}^{*}-\Omega_{c}$ & 0.071 & 0.086 & 0.089 & - & 0.086 & 0.10 \\
$\Xi_{c c}^{*}-\Xi_{c c}$ & - & 0.104 & 0.123 & - & 0.119 & - \\
$\Omega_{c c}^{*}-\Omega_{c c}$ & - & 0.090 & 0.106 & - & 0.100 & - \\
\hline
\end{tabular}

Table 4. Masses and mass splittings (in $\mathrm{GeV}$ ) for mesons belonging to the charm sector, calculated in various approaches as described in the text. The column denoted as Exp contains experimental data.

\begin{tabular}{|c|c|c|c|c|c|c|c|}
\hline Mesons & Quarks & Exp & Our & [16] & [49] & {$[50,51]$} & [34] \\
\hline$J / \psi$ & $c \bar{c}$ & 3.097 & 3.097 & 3.10 & 3.10 & 3.096 & 3.097 \\
\hline$\eta_{c}$ & $c \bar{c}$ & 2.980 & 3.005 & 2.97 & 3.00 & 2.979 & 2.990 \\
\hline$D$ & $c \bar{u}$ & 1.867 & 1.834 & 1.88 & 1.85 & 1.872 & 1.883 \\
\hline$D^{*}$ & $c \bar{u}$ & 2.008 & 2.002 & 2.04 & 2.02 & 2.009 & 2.010 \\
\hline$D_{s}$ & $c \bar{s}$ & 1.968 & 1.965 & 1.98 & 1.94 & 1.967 & 1.981 \\
\hline \multirow[t]{2}{*}{$D_{s}^{*}$} & $c \bar{s}$ & 2.112 & 2.119 & 2.13 & 2.13 & 2.112 & 2.112 \\
\hline & $\chi$ & - & 0.017 & 0.02 & 0.02 & 0.002 & 0.009 \\
\hline$J / \psi-\eta_{c}$ & $c \bar{c}$ & 0.117 & 0.092 & 0.13 & 0.10 & 0.117 & 0.107 \\
\hline$D^{*}-D$ & $c \bar{u}$ & 0.141 & 0.168 & 0.16 & 0.17 & 0.137 & 0.127 \\
\hline$D_{s}^{*}-D_{s}$ & $c \bar{s}$ & 0.144 & 0.154 & 0.15 & 0.19 & 0.145 & 0.131 \\
\hline
\end{tabular}

It is also useful to compare the results of our bag model calculations with the predictions obtained in other (including more elaborated) approaches. Let us start with the meson sector. In Tables 4 and 5 we compare our predictions for the masses of heavy mesons with the calculations in four different variants of the potential model. We have chosen for this the relativized quark model [16], the model based on the Bethe-Salpeter equation [49], the relativistic treatment using quasipotential approach $[50,51]$, and one specific variant of nonrelativistic potential model [34] where the Goldstone-boson exchanges are considered together with the one-gluon-exchange. The experimental values are from the Particle Data Tables [42] with the exeption of the $\eta_{b}$ meson. The mass value of $\eta_{b}$ is taken from [52].

As can be seen from these tables, the overall agreement of the heavy meson spectrum calculated in our work with the experimental data is quite good. Inspecting the meson mass differences we see that for the $D$ and $B$ mesons consisting of one heavy (charmed or bottom) and one light (up or down) quark the mass splitting obtained in our work is $\sim 20 \%$ too large. For the $D_{s}$ and $B_{s}$ mesons consisting of one heavy and one strange quark the agreement with experiment is better, while the predicted mass splitting is still too large. On the other hand, for the $c \bar{c}$ system the mass difference of $J / \psi$ and $\eta_{c}$ predicted in our model is about $30 \%$ too small, and we expect similar discrepancy for the $\Upsilon-\eta_{b}$. Regrettably, the discrepancy with experiment in this case is more severe. Our result is approximately three times smaller than experimental value, and this seems to be the serious drawback of the model. Since the $B_{c}$ meson is made of two heavy quarks, we expect that our prediction for the $B_{c}^{*}-B_{c}$ mass difference should be somewhat too small as well. So far, the $B_{c}$ meson is the only well-established system containing two different heavy quarks, and it naturally has attracted much attention these years. In Table 6 we have collected a number of predictions made by various authors for the ground state mass values of the $B_{c}$ and $B_{c}^{*}$ mesons.

Except for the lattice QCD prediction [8] given in the last column of this table and our estimate before it, all others are the potential model calculations. The numerical values of $B_{c}$ mass obtained in each model depend on the particular potential and vary in the interval from 6.24 to $6.29 \mathrm{GeV}$. Our prediction lies slightly higher and so does the lattice value. A possible reason 
Table 5. Masses and mass splittings (in $\mathrm{GeV}$ ) for mesons belonging to the bottom sector, calculated in various approaches as described in the text. The column denoted as Exp contains experimental data.

\begin{tabular}{|c|c|c|c|c|c|c|c|}
\hline Mesons & Quarks & Exp & Our & [16] & [49] & {$[50,51]$} & [34] \\
\hline$\Upsilon$ & $b \bar{b}$ & 9.460 & 9.460 & 9.46 & 9.46 & 9.460 & 9.505 \\
\hline$\eta_{b}$ & $b \bar{b}$ & 9.389 & 9.438 & 9.40 & 9.41 & 9.400 & 9.454 \\
\hline$B$ & $b \bar{u}$ & 5.279 & 5.249 & 5.31 & 5.28 & 5.275 & 5.281 \\
\hline$B^{*}$ & $b \bar{u}$ & 5.325 & 5.306 & 5.37 & 5.33 & 5.326 & 5.321 \\
\hline$B_{s}$ & $b \bar{s}$ & 5.367 & 5.383 & 5.39 & 5.37 & 5.362 & 5.355 \\
\hline$B_{s}^{*}$ & $b \bar{s}$ & 5.413 & 5.436 & 5.45 & 5.43 & 5.414 & 5.400 \\
\hline$B_{c}$ & $b \bar{c}$ & 6.276 & 6.304 & 6.27 & 6.26 & 6.270 & 6.277 \\
\hline \multirow[t]{2}{*}{$B_{c}^{*}$} & $b \bar{c}$ & - & 6.342 & 6.34 & 6.34 & 6.332 & - \\
\hline & $\chi$ & - & 0.027 & 0.03 & 0.01 & 0.005 & 0.031 \\
\hline$\Upsilon-\eta_{b}$ & $b \bar{b}$ & 0.071 & 0.022 & 0.06 & 0.05 & 0.060 & 0.051 \\
\hline$B^{*}-B$ & $b \bar{u}$ & 0.046 & 0.057 & 0.06 & 0.05 & 0.051 & 0.040 \\
\hline$B_{s}^{*}-B_{s}$ & $b \bar{s}$ & 0.046 & 0.053 & 0.06 & 0.06 & 0.052 & 0.045 \\
\hline$B_{c}^{*}-B_{c}$ & $b \bar{c}$ & - & 0.038 & 0.07 & 0.08 & 0.062 & - \\
\hline
\end{tabular}

Table 6. Comparison of various predictions for the masses (in $\mathrm{GeV}$ ) of $B_{c}$ and $B_{c}^{*}$ mesons.

\begin{tabular}{lcccccccc}
\hline & {$[53]$} & {$[54]$} & {$[55]$} & {$[51]$} & {$[56]$} & {$[57]$} & Our & {$[8]$} \\
\hline$B_{c}$ & 6.247 & 6.253 & 6.264 & 6.270 & 6.271 & 6.286 & 6.304 & 6.304 \\
$B_{c}^{*}$ & 6.308 & 6.317 & 6.337 & 6.332 & 6.338 & 6.341 & 6.342 & - \\
$B_{c}^{*}-B_{c}$ & 0.061 & 0.064 & 0.073 & 0.062 & 0.067 & 0.055 & 0.038 & - \\
\hline
\end{tabular}

for the mass of $B_{c}$ meson calculated in our model to be slightly higher than potential model predictions may be a supposed underestimate (look at the last row of Table 6) of the interaction strength for the heavy-heavy hadrons. Recent experimental results $6.276( \pm 6) \mathrm{GeV}$ [58] and $6.300( \pm 19) \mathrm{GeV}[59]$ cover the range from 6.270 to $6.319 \mathrm{GeV}$ and agree well with the theoretical estimates. The discovery of the $B_{c}$ meson and almost precise theoretical prediction of its mass is undoubtedly a great success of experiment and theory.

Let us proceed to the spectra of heavy baryons. We compare our predictions obtained in the modified bag model for baryons from the charm sector (Table 7) and for baryons from the bottom sector (Tables 8 and 9) with some other estimates and experimental data. The works we want to compare our results with are: the baryon mass estimates in relativistic $[29,30]$ and nonrelativistic $[25,26]$ potential models with assumed quark-diquark ansatz, usual nonrelativistic potential model [19], variational calculations [23, 24], and estimates obtained using various sum rules [60]. All but the one experimental masses for bottom baryons are from the Particle Data Tables [42]. The mass value for $\Omega_{b}$ is taken from Ref. [61].

From Table 7 we see that for baryons with one heavy quark ( $\Xi_{c}$ and $\Omega_{c}$ families) the predictions obtained in all approaches are in good agreement with experimental observations. The bag model predicts somewhat larger mass splittings for the states $\Xi_{c}^{*}-\Xi_{c}^{\prime}$ and $\Omega_{c}^{*}-\Omega_{c}$, but this does not spoil the fit substantially. For the corresponding baryons from the bottom sector presented in Table 8 , our predictions are in agreement with almost all other calculations again. So far there are only five bottom baryons observed $\left(\Lambda_{b}, \Sigma_{b}, \Sigma_{b}^{*}, \Xi_{b}\right.$, and $\left.\Omega_{b}\right)$. We see that all predictions for $\Xi_{b}$ compare favourably with the experimental data. For $\Sigma_{c}, \Sigma_{c}^{*}$ and $\Sigma_{b}, \Sigma_{b}^{*}$ baryons the bag model unfortunately is a bad adviser, while the potential model calculations fit well the experimental data again. The experimental mass value of the doubly strange $b$ baryon $\Omega_{b}$ lies $\sim 100 \mathrm{MeV}$ higher than expected from the theoretical estimates. All calculations in this case give similar results, and therefore it is hard to understand such discrepancy. The only thing we can say is that the $\Omega_{b}$ mass value observed in [61] still needs additional confirmation.

Another serious test of any model should be the ability to predict the masses of the baryons containing two heavy quarks. The only available candidate for this so far is the $\Xi_{c c}$ baryon, although the situation is not quite clear. The SELEX Collaboration has reported the observation of $\Xi_{c c}$ in two different experiments $[62,63]$. On the other hand, BABAR [64] and Belle [65] collaborations found no evidence of such baryon in their searches. From Table 7 we see that only two calcula- 
Table 7. Masses and mass splittings (in $\mathrm{GeV}$ ) of the charm sector baryons, calculated in modified bag model and in other approaches as described in the text. The column denoted as Exp contains experimental data.

\begin{tabular}{llccccccc}
\hline Baryons & Quarks & Exp & Our & {$[29,30]$} & {$[25,26]$} & {$[19]$} & {$[23,24]$} & {$[60]$} \\
\hline$\Lambda_{c}$ & $c u u$ & 2.286 & 2.286 & 2.297 & - & 2.268 & 2.295 & 2.285 \\
$\Sigma_{c}$ & $c u u$ & 2.454 & 2.393 & 2.439 & - & 2.455 & 2.469 & 2.453 \\
$\Sigma_{c}^{*}$ & $c u u$ & 2.518 & 2.489 & 2.518 & - & 2.519 & 2.548 & 2.530 \\
$\Xi_{c}$ & $c s u$ & 2.469 & 2.468 & 2.481 & - & 2.466 & 2.474 & 2.468 \\
$\Xi_{c}^{\prime}$ & $c s u$ & 2.577 & 2.546 & 2.578 & - & 2.594 & 2.578 & 2.582 \\
$\Xi_{c}^{*}$ & $c s u$ & 2.646 & 2.638 & 2.654 & - & 2.649 & 2.655 & 2.651 \\
$\Omega_{c}$ & $c s s$ & 2.697 & 2.697 & 2.698 & - & 2.718 & 2.681 & 2.710 \\
$\Omega_{c}^{*}$ & $c s s$ & 2.768 & 2.783 & 2.768 & - & 2.776 & 2.755 & 2.775 \\
$\Xi_{c c}$ & $c c u$ & 3.519 & 3.557 & 3.620 & 3.478 & 3.676 & 3.612 & 3.676 \\
$\Xi_{c c}^{*}$ & $c c u$ & - & 3.661 & 3.727 & 3.610 & 3.753 & 3.706 & 3.746 \\
$\Omega_{c c}$ & $c c s$ & - & 3.710 & 3.778 & 3.594 & 3.815 & 3.702 & 3.787 \\
$\Omega_{c c}^{*}$ & $c c s$ & - & 3.800 & 3.872 & 3.730 & 3.876 & 3.783 & 3.851 \\
$\Omega_{c c c}$ & $c c c$ & - & 4.777 & - & - & 4.965 & - & - \\
\cline { 3 - 9 } & & - & 0.027 & 0.008 & - & 0.012 & 0.015 & 0.007 \\
$\Sigma_{c}^{*}-\Sigma_{c}$ & $c u u$ & 0.064 & 0.096 & 0.079 & - & 0.064 & 0.079 & 0.077 \\
$\Xi_{c}^{*}-\Xi_{c}^{\prime}$ & $c s u$ & 0.069 & 0.092 & 0.076 & - & 0.055 & 0.077 & 0.069 \\
$\Omega_{c}^{*}-\Omega_{c}$ & $c s s$ & 0.071 & 0.086 & 0.070 & - & 0.058 & 0.074 & 0.065 \\
$\Xi_{c c}^{*}-\Xi_{c c}$ & $c c u$ & - & 0.104 & 0.107 & 0.132 & 0.077 & 0.094 & 0.070 \\
$\Omega_{c c}^{*}-\Omega_{c c}$ & $c c s$ & - & 0.090 & 0.094 & 0.136 & 0.061 & 0.081 & 0.064 \\
\hline
\end{tabular}

Table 8. Masses (in $\mathrm{GeV}$ ) of the bottom sector baryons, calculated in modified bag model and in other approaches as described in the text. The column denoted as Exp contains experimental data.

\begin{tabular}{llrlrrrrr}
\hline Baryons & Quarks & Exp & \multicolumn{1}{c}{ Our } & {$[29,30]$} & {$[25,26]$} & {$[19]$} & {$[23,24]$} & \multicolumn{1}{c}{$[60]$} \\
\hline$\Lambda_{b}$ & $b u u$ & 5.620 & 5.620 & 5.622 & - & 5.612 & 5.643 & 5.627 \\
$\Sigma_{b}$ & $b u u$ & 5.811 & 5.755 & 5.805 & - & 5.833 & 5.851 & 5.818 \\
$\Sigma_{b}^{*}$ & $b u u$ & 5.833 & 5.787 & 5.834 & - & 5.858 & 5.882 & 5.843 \\
$\Xi_{b}$ & $b s u$ & 5.792 & 5.809 & 5.812 & - & 5.806 & 5.808 & - \\
$\Xi_{b}^{\prime}$ & $b s u$ & - & 5.911 & 5.937 & - & 5.970 & 5.946 & 5.955 \\
$\Xi_{b}^{*}$ & $b s u$ & - & 5.944 & 5.963 & - & 5.980 & 5.975 & 5.984 \\
$\Omega_{b}$ & $b s s$ & 6.165 & 6.067 & 6.065 & - & 6.081 & 6.033 & 6.075 \\
$\Omega_{b}^{*}$ & $b s s$ & - & 6.096 & 6.088 & - & 6.102 & 6.063 & 6.098 \\
$\Xi_{b c}$ & $b c u$ & - & 6.846 & 6.933 & 6.82 & 7.011 & 6.919 & 7.029 \\
$\Xi_{b c}^{\prime}$ & $b c u$ & - & 6.891 & 6.963 & 6.85 & 7.047 & 6.948 & 7.053 \\
$\Xi_{b c}^{*}$ & $b c u$ & - & 6.919 & 6.980 & 6.90 & 7.074 & 6.986 & 7.083 \\
$\Omega_{b c}^{\prime}$ & $b c s$ & - & 6.999 & 7.088 & 6.93 & 7.136 & 6.986 & 7.126 \\
$\Omega_{b c}^{\prime}$ & $b c s$ & - & 7.036 & 7.116 & 6.97 & 7.165 & 7.009 & 7.148 \\
$\Omega_{b c}^{*}$ & $b c s$ & - & 7.063 & 7.130 & 7.00 & 7.187 & 7.046 & 7.165 \\
$\Omega_{b c c}^{*}$ & $b c c$ & - & 7.984 & - & - & 8.245 & - & - \\
$\Omega_{b c c}^{*}$ & $b c c$ & - & 8.005 & - & - & 8.265 & - & - \\
$\Xi_{b b}$ & $b b u$ & - & 10.062 & 10.202 & 10.093 & 10.340 & 10.197 & - \\
$\Xi_{b b}^{*}$ & $b b u$ & - & 10.101 & 10.237 & 10.133 & 10.367 & 10.236 & 10.398 \\
$\Omega_{b b}^{*}$ & $b b s$ & - & 10.208 & 10.359 & 10.210 & 10.454 & 10.260 & - \\
$\Omega_{b b}^{*}$ & $b b s$ & - & 10.244 & 10.389 & 10.257 & 10.486 & 10.297 & 10.483 \\
$\Omega_{b b c}$ & $b b c$ & - & 11.139 & - & 11.12 & 11.535 & - & - \\
$\Omega_{b b c}^{*}$ & $b b c$ & - & 11.163 & - & 11.18 & 11.554 & - & - \\
$\Omega_{b b b}$ & $b b b$ & - & 14.276 & - & - & 14.834 & - & - \\
\hline & & & & & & & & \\
\hline
\end{tabular}


Table 9. Mass splittings (in GeV) of the bottom sector baryons, calculated in modified bag model and in other approaches as described in the text.

\begin{tabular}{lcccccc}
\hline Baryons & Our & {$[29,30]$} & {$[25,26]$} & {$[19]$} & {$[23,24]$} & {$[60]$} \\
\hline$\Sigma_{b}^{*}-\Sigma_{b}$ & 0.032 & 0.029 & - & 0.025 & 0.031 & 0.025 \\
$\Xi_{b}^{*}-\Xi_{b}^{\prime}$ & 0.033 & 0.026 & - & 0.010 & 0.029 & 0.029 \\
$\Omega_{b}^{*}-\Omega_{b}$ & 0.029 & 0.023 & - & 0.021 & 0.030 & 0.023 \\
$\Xi_{b c}^{*}-\Xi_{b c}^{\prime}$ & 0.028 & 0.017 & 0.050 & 0.027 & 0.038 & 0.030 \\
$\Omega_{b c}^{*}-\Omega_{b c}^{\prime}$ & 0.027 & 0.014 & 0.030 & 0.022 & 0.037 & 0.017 \\
$\Omega_{b c c}^{*}-\Omega_{b c c}$ & 0.021 & - & - & 0.020 & - & - \\
$\Xi_{b b}^{*}-\Xi_{b b}$ & 0.039 & 0.035 & 0.040 & 0.027 & 0.039 & - \\
$\Omega_{b b}^{*}-\Omega_{b b}$ & 0.036 & 0.030 & 0.047 & 0.032 & 0.037 & - \\
$\Omega_{b b c}^{*}-\Omega_{b b c}$ & 0.024 & - & 0.060 & 0.019 & - & - \\
\hline
\end{tabular}

tions ([25] and ours) predict $\Xi_{c c}$ mass compatible with the SELEX result. All other approaches predict the mass of this baryon to be $100-150 \mathrm{MeV}$ higher. If we accept the SELEX result as true (regardless of the absence of independent confirmations), this would be the second doubly-heavy hadron observed (the first was $B_{c}$ meson). In both cases the predictions of the modified bag model are sufficiently good. The comparison of our results with others shows that for doublyheavy baryons all approaches give qualitatively similar (the same ordering of states) predictions, while the numerical values could differ substantially. The lowest mass values are obtained in Refs. [25, 26], our values lie higher by about 70-80 MeV, and all other approaches predict even larger masses of these baryons. As regards the mass splittings of doubly-heavy baryons $\Xi_{c c}^{*}-\Xi_{c c}$ and $\Omega_{c c}^{*}-\Omega_{c c}$, the predictions vary from about $70 \mathrm{MeV}$ [60] to $130 \mathrm{MeV}$ [26], our estimate (approximately 100 $\mathrm{MeV}$ ) together with the predictions given by Ref. [29] being somewhere in the middle. For the triple-heavy baryon $\Omega_{c c c}$ the bag model predicted mass is about 200 $\mathrm{MeV}$ smaller than the corresponding mass value in nonrelativistic potential model [19].

We expect the predicted spectrum of the bottom sector baryons to be qualitatively similar to the spectrum in the charm sector, and indeed we find similar regularities in the spectrum of bottom baryons. For the doubly-heavy baryons containing heavy quarks of distinct flavour (charm and bottom) the lowest baryon masses are predicted in Refs. [25,26]. For the baryons from the $\Xi_{b c}$ family our predictions are similar to [25, 26], while the predictions of Refs. [29] and [24] are approximately $80 \mathrm{MeV}$ higher than ours, and predictions of Refs. [19] and [60] exceed ours by about $170 \mathrm{MeV}$. For the $\Omega_{b c}$ family our results are similar to the calculations of Ref. [24] and about $60 \mathrm{MeV}$ above the values obtained in $[25,26]$. The largest masses (approximately $120 \mathrm{MeV}$ higher than ours) are again given by [19] and
[60]. For the baryons containing two bottom quarks ( $\Xi_{b b}$ and $\Omega_{b b}$ families) our calculations predict the lowest baryon mass values. Predictions of Refs. [25, 26] are also very close to ours. The largest masses in this case (about $240-280 \mathrm{MeV}$ higher then ours) are given by [19]. For the triply-heavy baryons containing one bottom and two charmed quarks ( $\Omega_{b c c}$ family) Ref. [19] predicts the baryon masses $260 \mathrm{MeV}$ higher than ours, the result similar as in the case of $\Xi_{b b}$ and $\Omega_{b b}$ baryons. Predictions for the masses of the triply-heavy baryons containing two bottom and one charmed quark $\left(\Omega_{b b c}\right.$ family) in our model and in Refs. [25,26] are similar again, and mass values from Ref. [19] are commonly higher than ours (in this case by about $400 \mathrm{MeV}$ ). The largest difference between predictions of Ref. [19] and baryon mass values calculated in our model $(560 \mathrm{MeV})$ is obtained for the heaviest ground state baryon $\Omega_{b b b}$. As regards the predictions for the baryon mass splittings, the situation in the bottom sector is evidently simpler, and almost all approaches give similar results. The reason is also clear: the interaction in this case is much weaker and consequently it causes smaller mass splittings. Because the heavier systems seem to be simpler, one could naively expect that in such case all reasonable approaches give similar predictions. As we have seen from Tables 7 and 8, in general this is not the case. Moreover, the situation with theoretical predictions of the heavy baryon masses seems to be controversial to some extent. For the baryons containing only one heavy quark all approaches, as a rule, give similar predictions in reasonable agreement with available experimental data. On the other hand, in the case of doublyheavy (as well as triply-heavy) baryons the predicted values strongly depend on the model. Sometimes very different approaches (e. g., Refs. [25, 26] and our modified bag model) give very similar predictions, however, this is rather an exception than a rule. Evidently, further investigations in this field are necessary. On the 
other hand, the rapid development of experimental and theoretical methods in recent years is rather impressive, and we expect that in the nearest future new experimental data and new improved results of lattice calculations will shed some light on the subject.

\section{Conclusions and discussion}

In conclusion, we have calculated the spectrum of all ground state baryons containing bottom quarks by means of a modified bag model suitable for the unified description of heavy and light hadrons. The model parameters are practically the same as in our previous paper [39] (following the procedure proposed in the original MIT bag version the main four of them have been determined from the light hadrons). For completeness the calculated masses for the hadrons belonging to the charm sector are also presented. All the predictions are compared with the calculations in other approaches and with experimental data where available. For mesons and baryons containing one heavy quark the agreement is good. Therefore we expect our predictions for the doubly-heavy (and, maybe, triply-heavy) baryon masses to be useful complementary tool in the treatment of heavy baryon spectra.

Regretfully, the predictional power of the bag model is not so high, as, for example, potential model in its various incarnations. The annoying thing is that the applications of the bag model practically are restricted to the calculation of properties of just the ground state hadrons. There was some work done to incorporate the excited states in various versions (usual MIT bag, chiral bag, etc.) of the model [66-69]. In general the attempts were rather successful, however, the complications associated with relativity and the problem of spurious centre-of-mass motion makes the bag model in this case calculationally much more unwieldy than the nonrelativistic models. On the other hand, in some cases the initial simplicity of the model may be regarded as an advantage, and old-good bag model could serve for a while as a modest but still useful tool for investigation of various hadronic properties (ground state hadron masses, magnetic moments, isospin splittings, etc.), especially when the preliminary quick estimate is necessary.

\section{References}

[1] J.L. Rosner, J. Phys. G 34 (2007) S127.

[2] K.C. Bowler et al. (UKQCD Collaboration), Phys. Rev. D 54 (1996) 3619.
[3] A. Ali Khan et al., Phys. Rev. D 62 (2000) 054505.

[4] N. Mathur, R. Lewis, and R.M. Woloshyn, Phys. Rev. D 66 (2002) 014502.

[5] J.M. Flynn, F. Mescia, and A.S.B. Tariq, J. High Energy Phys. 07 (2003) 066.

[6] T. Chiu and T. Hsieh, Nucl. Phys. A 755 (2005) 471.

[7] H. Na and S. Gottlieb, PoS (LATTICE 2007) 190 [arXiv: 0710.1422].

[8] I.F. Allison et al. (HPQCD, Fermilab Lattice, and UKQCD Collaborations), Phys. Rev. Lett. 94 (2005) 172001.

[9] F.O. Duraes and M. Nielsen, Phys. Lett. B 658 (2007) 40.

[10] X. Liu, H. Chen, Y. Liu, A. Hosaka, and S. Zhu, Phys. Rev. D 77 (2008) 014031.

[11] J.G. Körner, M. Krämer, and D. Pirjol, Prog. Part. Nucl. Phys. 33 (1994) 787.

[12] M. Neubert, Phys. Rept. 245 (1994) 259.

[13] T. Mannel, Rep. Prog. Phys. 60 (1997) 1113.

[14] I. Bigi, M. Shifman, and N. Uraltsev, Ann. Rev. Nucl. Part. Sci. 47 (1997) 591.

[15] L.A. Copley, N. Isgur, and G. Karl, Phys. Rev. D 20 (1979) 768 [Erratum - ibid. 23 (1991) 817].

[16] S. Godfrey and N. Isgur, Phys. Rev. D 32 (1985) 189.

[17] S. Capstic and N. Isgur, Phys. Rev. D 34 (1986) 2809.

[18] M. Pervin and W. Roberts, Phys. Rev. C 77 (2008) 025202.

[19] W. Roberts and M. Pervin, Int. J. Mod. Phys. A 23 (2008) 2817.

[20] F. Coester, K. Dannbom, and D.O. Riska, Nucl. Phys. A 634 (1998) 335.

[21] H. Garcilazo, J. Vijande, and A. Valcarce, J. Phys. G 34 (2007) 961.

[22] C. Albertus, J.E. Amaro, E. Hernández, and J. Nieves, Nucl. Phys. A 740 (2004) 333.

[23] C. Albertus, J.E. Amaro, E. Hernández, and J. Nieves, Nucl. Phys. A 755 (2005) 439.

[24] C. Albertus, E. Hernández, J. Nieves, and J.M. VerdeVelasco, Eur. Phys. J. A 31 (2007) 691.

[25] S.S. Gershtein, V.V. Kiselev, A.K. Likhoded, and A.I. Onishchenko, Phys. Rev. D 62 (2000) 054021.

[26] V.V. Kiselev, A.K. Likhoded, O.N. Pakhomova, and V.A. Saleev, Phys. Rev. D 66 (2002) 034030.

[27] S. Migura, D. Merten, B. Metsch, and H. Petry, Eur. Phys. J. A 28 (2006) 41.

[28] D. Ebert, R.N. Faustov, V.O. Galkin, A.P. Martynenko, and V.A. Saleev, Z. Phys. C 76 (1997) 111.

[29] D. Ebert, R.N. Faustov, V.O. Galkin, and A.P. Martynenko, Phys. Rev. D 66 (2002) 014008.

[30] D. Ebert, R.N. Faustov, and V.O. Galkin, Phys. Rev. D 72 (2005) 034026.

[31] A. De Rújula, H. Georgi, and S.L. Glashow, Phys. Rev. D 12 (1975) 147.

[32] L.Ya. Glozman and D.O. Riska, Nucl. Phys. A 603 (1996) 326 [Erratum - ibid. 620 (1997) 510]. 
[33] L.Ya. Glozman, W. Plessas, K. Varga, and R.F. Wagenbrunn, Phys. Rev. D 58 (1998) 094030.

[34] J. Vijande, F. Fernández, and A. Valcarce, J. Phys. G 31 (2005) 481.

[35] T. DeGrand, R.L. Jaffe, K. Johnson, and J. Kiskis, Phys. Rev. D 12 (1975) 2060.

[36] R.L. Jaffe and J. Kiskis, Phys. Rev. D 13 (1976) 1355.

[37] W.A. Ponce, Phys. Rev. D 19 (1979) 2197.

[38] D. Izatt, C. DeTar, and M. Stephenson, Nucl. Phys. B 199 (1982) 269.

[39] A. Bernotas and V. Šimonis, Nucl. Phys. A. 741 (2004) 179.

[40] L.C.L. Hollenberg and B.H.J. McKellar, J. Phys. G 16 (1990) 31.

[41] J.F. Donoghue and K. Johnson, Phys. Rev. D 21 (1980) 1975.

[42] C. Amsler et al. (Particle Data Group), Phys. Lett. B 667 (2008) 1.

[43] J. Franklin, D.B. Lichtenberg, W. Namgung, and D. Carydas, Phys. Rev. D 24 (1981) 2910.

[44] A. Bernotas and V. Šimonis, Lithuanian J. Phys. 48 (2008) 127.

[45] J. Schaffner-Bielich and A.P. Vischer, Phys. Rev. D 57 (1998) 4142.

[46] F. Myhrer, G.E. Brown, and Z. Xu, Nucl. Phys. A 362 (1981) 317.

[47] P.J. Mulders and A.W. Thomas, J. Phys. G 9 (1983) 1159.

[48] K. Saito, Prog. Theor. Phys. 71 (1984) 775.

[49] J. Zeng, J.W. Van Orden, and W. Roberts, Phys. Rev. D 52 (1995) 5229.

[50] D. Ebert, R.N. Faustov, and V.O. Galkin, Phys. Lett. B 635 (2006) 93.
[51] D. Ebert, R.N. Faustov, and V.O. Galkin, Phys. Rev. D 67 (2003) 014027.

[52] B. Aubert et al. (BABAR Collaboration), Phys. Rev. Lett. 101 (2008) 071801.

[53] S.N. Gupta and J.M. Johnson, Phys. Rev. D 53 (1996) 312.

[54] S.S. Gershtein, V.V. Kiselev, A.K. Likhoded, and A.V. Tkabladze, Phys. Rev. D 51 (1995) 3613.

[55] E.J. Eichten and C. Quigg, Phys. Rev. D 49 (1994) 5845 .

[56] S. Godfrey, Phys. Rev. D 70 (2004) 054017.

[57] L.P. Fulcher, Phys. Rev. D 60 (1999) 074006.

[58] T. Aaltonen et al. (CDF Collaboration), Phys. Rev. Lett. 100 (2008) 182002.

[59] V.M. Abazov et al. (DØ Collaboration), Phys. Rev. Lett. 101 (2008) 012001.

[60] D.B. Lichtenberg, R. Roncaglia, and E. Predazzi, Phys. Rev. D 53 (1996) 6678.

[61] V.M. Abazov et al. (DØ Collaboration), Phys. Rev. Lett. 101 (2008) 232002.

[62] M. Mattson et al. (SELEX Collaboration), Phys. Rev. Lett. 89 (2002) 112001.

[63] A. Ocherashvili et al. (SELEX Collaboration), Phys. Lett. B 628 (2005) 18.

[64] B. Aubert et al. (BABAR Collaboration), Phys. Rev. D 74 (2006) 011103(R).

[65] R. Chistov et al. (Belle Collaboration), Phys. Rev. Lett. 97 (2006) 162001.

[66] T.A. DeGrand, Ann. Phys. (N.Y.) 101 (1976) 496.

[67] F. Myhrer and J. Wroldsen, Phys. Lett. B 139 (1984) 81.

[68] Y. Umino and F. Myhrer, Phys. Rev. D 39 (1989) 3391.

[69] A.H. Örsland and H. Högaasen, Eur. Phys. J. C 9 (1999) 503.

\title{
SUNKIŨJŲ BARIONŲ SPEKTROSKOPIJA IR MAIŠŲ MODELIS
}

\author{
A. Bernotas, V. Šimonis \\ Vilniaus universiteto Teorinės fizikos ir astronomijos institutas, Vilnius, Lietuva
}

\begin{abstract}
Santrauka
Taikant modifikuotą MIT maišų modelį, apskaičiuotos sunkiųjų hadronų, i kurių sudètį įeina „sunkūs“ $c$ ir $b$ kvarkai, pagrindinių būsenų masès. Gautos darbe masių vertès palygintos su kitų autoriu i̇vairiuose modeliuose atliktų skaičiavimų rezultatais bei turimais
\end{abstract}

naujausiais eksperimentų duomenimis. Daugeliu atvejų sutapimas su eksperimentu bei kitais skaičiavimais yra neblogas. Taip pat nurodyti kai kurie modelio trūkumai, aptarti privalumai bei modelio vieta teorinejje hadronų spektroskopijoje. 